REVIEW ARTICLE

https://doi.org/10.1038/s41467-019-08981-w

OPEN

\title{
Halide lead perovskites for ionizing radiation detection
}

\author{
Haotong Wei (i) ${ }^{1} \&$ Jinsong Huang (i) ${ }^{1}$
}

Halide lead perovskites have attracted increasing attention in recent years for ionizing radiation detection due to their strong stopping power, defect-tolerance, large mobilitylifetime $(\mu \tau)$ product, tunable bandgap and simple single crystal growth from low-cost solution processes. In this review, we start with the requirement of material properties for high performance ionizing radiation detection based on direct detection mechanisms for applications in $\mathrm{X}$-ray imaging and $\boldsymbol{\gamma}$-ray energy spectroscopy. By comparing the performances of halide perovskites radiation detectors with current state-of-the-art ionizing radiation detectors, we show the promising features and challenges of halide perovskites as promising radiation detectors.

\footnotetext{
${ }^{1}$ Department of Applied Physical Sciences, University of North Carolina, Chapel Hill, NC 27599, USA. Correspondence and requests for materials should be addressed to J.H. (email: jhuang@unc.edu)
} 
T he strong penetrating capability of high-energy ionizing radiation, including X-ray (photon with wavelength from 0.01 to $10 \mathrm{~nm})$ or gamma-ray ( $\gamma$-ray, photon with wavelength shorter than $0.01 \mathrm{~nm}$ ), make them attractive in nondestructive probing of inside information of condensed matter subjects. After their interaction with electrons in the atoms to ionize the materials, $\mathrm{X}$-rays and $\gamma$-rays carry information of the subjects in their variation of the photon intensity (dose rate), direction, phase, and energy. The detection of the X- and $\gamma$-ray variation in good faith thus allows accurate retrieval of the subject information. This enables the broad applications of X-ray in many fields including medical imaging, safety screening, shipping container inspection, defect inspection and quality control in material industry, and contamination detection and quality control inspection in food industry, where X-rays penetrate subjects and its attenuation is recorded ${ }^{1-5}$. The image contrast comes from the difference in absorption or stopping capability of different human tissues or subjects to X-rays. In scientific research, X-rays have been broadly used to enhance the understanding of material sciences in the past few decades by the interaction of X-rays with materials. $\gamma$-Rays can be generated in radioactive materials, which presents fingerprints of elements as well as isotopes, and distinguishing these elements or isotopes needs the detection of the energy-dependent photon counts, or spectroscopy. $\gamma$-Ray detection is essential for many homeland security applications such as radiological security, nuclear defense, and so on ${ }^{6-9}$.

Semiconductor-based ionizing radiation detectors work in a similar way with photodetectors either in current or voltage modes, while more details of specific difference are described in Box $1^{10,11}$. The current advanced radiation detectors working in current mode include silicon ( $\mathrm{Si}$ ), amorphous selenium ( $\alpha$-Se), mercury(II) iodide $\left(\mathrm{HgI}_{2}\right)$, cadmium zinc telluride $\left(\mathrm{Cd}_{1-}\right.$

\section{Box 1 | X- and $\gamma$-ray direct detection mechanism}

X-ray detectors for dosimeter or medical imaging work in current mode, and the photon flux is relatively strong to get enough signal/noise ratio, smooth image, and fast frame rate. Multi-photons come to the semiconductor detector together, and one X-ray photon can be directly converted into many excess free charges within the semiconductors through photoelectric effect process, which are then collected by electrodes under bias as shown in Box 1. In Compton scattering process, the incident photons will be deflected by electrons with a scattering angle with respect to the incident direction, and pass part of their energy to electrons depending on the photon energy and scattering angle. Many charges are generated in these two processes at the same time when attenuation occurs, which will be collected by the electrodes under bias to product signal current. All the attenuated X-ray photons contribute to the average signal current per unit time. The transmitted photon flux intensity $(I)$ follows a relationship of $I=I_{0} \times e^{-\alpha \rho l}$, where $I_{0}$ is the original photon flux intensity, $\alpha$ is the mass attenuation coefficient, $\rho$ is the materials density, and $I$ is the interaction length with materials. The linear attenuation coefficient $\alpha \rho$, also expressed as $\mu_{0}$, is often used to characterize the stopping power of a material.

While the radiation detectors for photon counters, spectroscopy, and nuclear reaction monitor often work in voltage mode. In contrast to the current mode, the photon flux intensity in voltage mode is relatively weak, and high-energy photons come to a detector one by one. Each photon will generate some electron-hole pairs, and the number of corresponding charge is counted in the signal pulse, the intensity of which is proportional to the photon energy as shown in Box 1, voltage mode. By histogramming the photon energy one after another, a $\gamma$-ray photon energy spectrum can be plotted. In order to distinguish the small signal, a charge-sensitive preamplifier is often employed to integrate the signal coming from the detector, thus converting the collected charge into a voltage signal. A quasi-Gaussian output is then performed in a connected shaping amplifier, reshaping the output signal peak to make the transmitted signal better suited to the communication channel, whose intensity is proportional to the photon energy. Finally, a multichannel analyzer can count the radiation quanta versus energy, and generate $X$ - or $\gamma$-ray energy spectrum by recording photons one by one ${ }^{75,76}$

\section{Current mode}
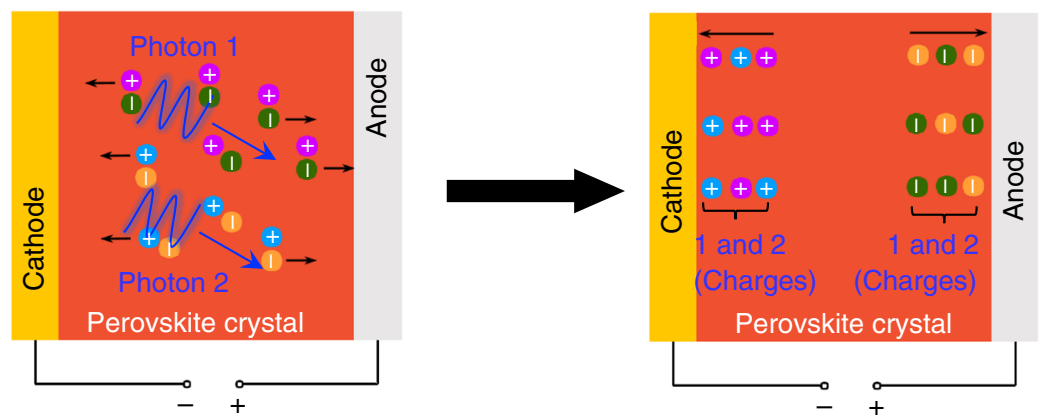

Voltage mode
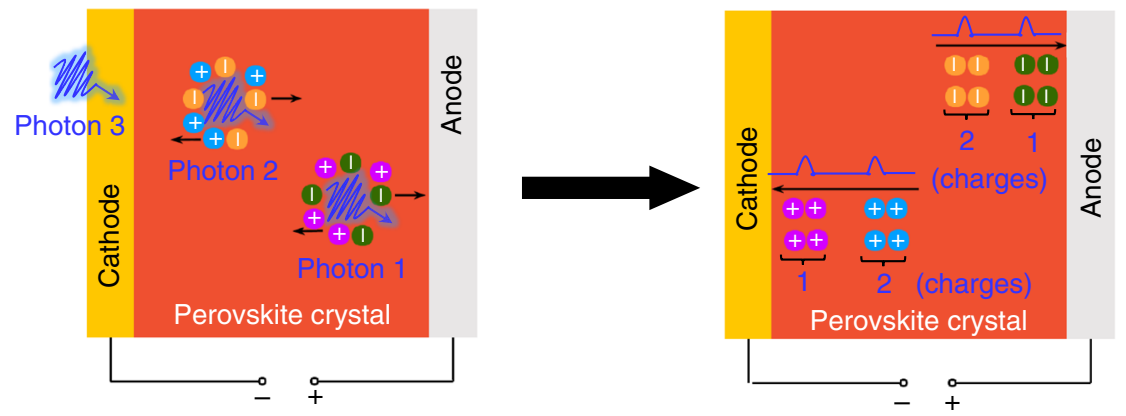
${ }_{x} \mathrm{Zn}_{x} \mathrm{Te}$ ), while radiation detectors in voltage mode comprise high-purity germanium (HPGe), $\mathrm{Cd}_{1-}{ }_{x} \mathrm{Zn}_{x} \mathrm{Te}$, thallium(I) bromide (TlBr), and so on. Besides direct detection strategy, $\mathrm{X}$ - and $\gamma$-ray detection can also be accomplished in an indirect detection strategy through a combination of scintillators and photodetectors. Scintillators convert high-energy X-rays and $\gamma$-rays into ultraviolet-visible (UV-Vis) light, which is detected by weak light sensors such as amorphous Si photodiodes, thin film phototransistor (TFT) arrays, photomultiplier tubes (PMTs), complementary metal-oxide semiconductor (CMOS) detectors, silicon avalanche photodiodes and arrays, or charge-coupled devices (CCDs). The dominating commercial X-ray and $\gamma$-ray scintillators include thallium-activated cesium iodide (CsI(Tl)), thalliumactivated sodium iodide $(\mathrm{NaI}(\mathrm{Tl})$ ), and so on. Important performance parameters for scintillators are light yield, light decay time, and environmental stability.

The explosion in the use of higher radiation-dose tests, such as computed tomography (CT) imaging, brings concerns on radiation safety. Over 80 million CT scans are performed in the United States, compared with just 3 million in 1980. High-dose X-ray scan increases the risk of cancer to patient later in life due to the damaging of DNA by ionizing radiation ${ }^{12}$. For example, one-time chest CT scan can have dose equivalent to 2 years accumulation of natural background dose ${ }^{12}$. There is a report showing evidence of radiation-induced cancer risk at a dose above $100 \mathrm{mSv}$, as well as an estimation of $\sim 2 \%$ of the cancers diagnosed could be related to CT performed in the United States in $2007^{12}$. The high dose needed for CT scanning comes from the low sensitivity of the present radiation detectors, mainly limited by the detection properties of materials. Therefore, high-performance radiation detectors based on new materials are urgently needed.

In the past few years, a new family of halide perovskites with composition of $\mathrm{ABX}_{3}$ (where $\mathrm{A}$ is $\mathrm{CH}_{3} \mathrm{NH}_{3}{ }^{+}\left(\mathrm{MA}^{+}\right), \mathrm{HN}=$ $\mathrm{CHNH}_{3}^{+}\left(\mathrm{FA}^{+}\right)$, or $\mathrm{Cs}^{+} ; \mathrm{B}$ is $\mathrm{Pb}^{2+}, \mathrm{Sn}^{2+} ; \mathrm{X}$ is $\mathrm{Cl}^{-}, \mathrm{Br}^{-}$, and $\left.\mathrm{I}^{-}\right)$ or $\mathrm{A}_{2} \mathrm{MM}^{\prime} \mathrm{X}_{6}$ (where $\mathrm{M}$ is $\mathrm{Cu}^{+}$, or $\mathrm{Ag}^{+}, \mathrm{M}^{\prime}$ is $\mathrm{Bi}^{3+}, \mathrm{Ga}^{3+}$, or $\mathrm{In}^{3+}$ ) have attracted lots of attention by their excellent performance in solar cells, light-emitting diodes, lasers, and photodetectors during the past few years ${ }^{13-16}$. Halide perovskites are quickly identified to be promising candidate semiconductors for ionizing radiation detection ${ }^{17-19}$. This review exploits the science and application of halide perovskites as a new semiconductor candidate for X-ray imaging and $\gamma$-ray spectroscopy. Although there are multiple-stringent requirements on ionizing detector properties, which results in very limited choice on effective materials, halide perovskites show many advantages as promising radiation detector materials, which are discussed in detail in the following sections. There are still outstanding challenges before their commercialization, such as how to stabilize material and interface under large bias in single-photon detectors, which will also be discussed in this review.

\section{Development status of conventional radiation detectors} $\mathrm{X}$-ray dosimeter and imaging detectors by direct conversion. For X-ray imaging detectors, a large sensitivity is needed for highquality images with large contrast at a given X-ray dose rate ${ }^{20}$, which outputs larger X-ray-induced current ${ }^{10,21}$. In order to minimize the doses applied to the patient while maintaining image quality, one path is to increase the mobility-lifetime $(\mu \tau)$ product or increase the applied bias to the detectors ${ }^{22}$.

The $\mu \tau$ product, which characterizes carrier drift length, is a fundamental property of a semiconductor material to predict the capability of extracting the charges deep inside the semiconductor. The $\mu \tau$ product can be determined by material types as well as defects, which is a parameter closely related to the charge carrier diffusion length $L_{\mathrm{D}}$ with a relationship of $L_{\mathrm{D}}=\left(k_{\mathrm{B}} T \mu \tau / e\right)^{1 / 2}$, where $k_{\mathrm{B}}, T$, and $e$ are the Boltzmann constant, absolute temperature, and elementary charge, respectively. Larger $\mu \tau$ product will result in better charge collection efficiency by increasing the mean drift lengths and/or diffusion lengths in the semiconductor bulk. Applying a high bias can enhance the signal intensity; however, it also increases the device noise.

In addition to a strong signal, a small noise is also important for a large signal-to-noise ratio, which is a figure of merit for many types of sensors ${ }^{18,19,21}$. The noise level also determines the weakest X-ray dose a detector can measure. The noise of a radiation detector is generally dominated by dark current, though other types of noise might also be present. While some other figures of merit are also important for a high-quality X-ray image, including spatial resolution, response speed, uniformity, and stability. Spatial resolution is a parameter used to characterize image sharpness, which can be quantitatively assessed by the modulation transfer function (MTF ${ }^{23}$. Linear dynamic range (LDR) is another important parameter for an X-ray detector to show the responsible range of X-ray dose rate under which sensitivity is a constant. Large LDR will ensure that the detector gives an accurate measurement of X-ray dose rate under a large variation range. Moreover, the $\mathrm{X}$-ray detector needs to have a fast response speed to reduce the exposure time of $\mathrm{X}$-ray irradiation, and also give high frame rate for capturing videos. Frame rate is the inverse of the time needed for the CCD to acquire an image and then completely read an image out, which is not only related to device response time but also the read-out circuitry. A uniform performance of the pixels among large area is also crucial for a high-quality X-ray imager, though it can be partially made-up with imaging processing.

Device performance of conventional X-ray detectors. Table 1 summarizes the detailed performance of the current X-ray detectors. They are made by $\mathrm{Si}, \boldsymbol{\alpha}-\mathrm{Se}, \mathrm{HgI}_{2}, \mathrm{Cd}_{1-x} \mathrm{Zn}_{x} \mathrm{Te}$, and so on. Since Si has low stopping power to X-ray, it is mainly applied in portable X-ray fluorescence spectrometry for soft X-ray detection, especially for photon energy smaller than $10 \mathrm{keV}$ to detect the fingerprints of trace elements as well as isotopes of different bulk materials ${ }^{24}$. Since $\alpha$-Se has over ten times stronger stopping power than $\mathrm{Si}$ in hard X-ray range ${ }^{25,26}$, it is the most common semiconductor material for X-ray imaging in direct conversion mode, and it has dominated the direct conversion Xray imaging market for decades ${ }^{10}$. Although the cost of $a$-Se raw material is only $\sim \$ 0.8$ per $\mathrm{g}$, the main flat-panel detector cost comes from thick film deposition process by conventional vacuum thermal deposition. $\mathrm{HgI}_{2}$ and $\mathrm{Cd}_{1-x} \mathrm{Zn}_{x} \mathrm{Te}(x$ is $<20 \%$, denoted as CZT) are both promising X-ray detectors. Their sensitivities are good as shown in Table 1, but the performance of $\mathrm{HgI}_{2}$ detector is mainly limited by the large leakage current. While there are also challenges in integrating CZT with read-out circuitry due to the too high temperature that required for highquality CZT crystal growth.

The current state-of-the-art CsI(Tl) scintillator detectors with CMOS sensor have pixel resolution around $50 \mu \mathrm{m}$, or $10 \mathrm{lpmm}^{-1}$ (ref. ${ }^{27}$ ), and corresponding X-ray sensitivity ${ }^{28}$ reaches $5370 \mu \mathrm{C}$ $\mathrm{Gy}^{-1}$ air $\mathrm{cm}^{-2}$. A recent reported scintillator made by terbiumdoped gadolinium oxysulfide (GOS:Tb) has been employed for Xray imaging with spatial resolution of $4.75 \mathrm{lp} \mathrm{mm}^{-1}$ (ref. ${ }^{29}$ ). Although the resolution is not high and the sensitivity is only about $7.3 \mu \mathrm{CGy}^{-1}$ air $\mathrm{cm}^{-2}$, GOS:Tb scintillator are still attractive for its low-cost fabrication from solution.

$\gamma$-Ray spectroscopy detectors. As for the $\gamma$-ray counting for energy spectrum application, the most important figure of merit is the photopeak energy resolution, which is defined by the ratio 

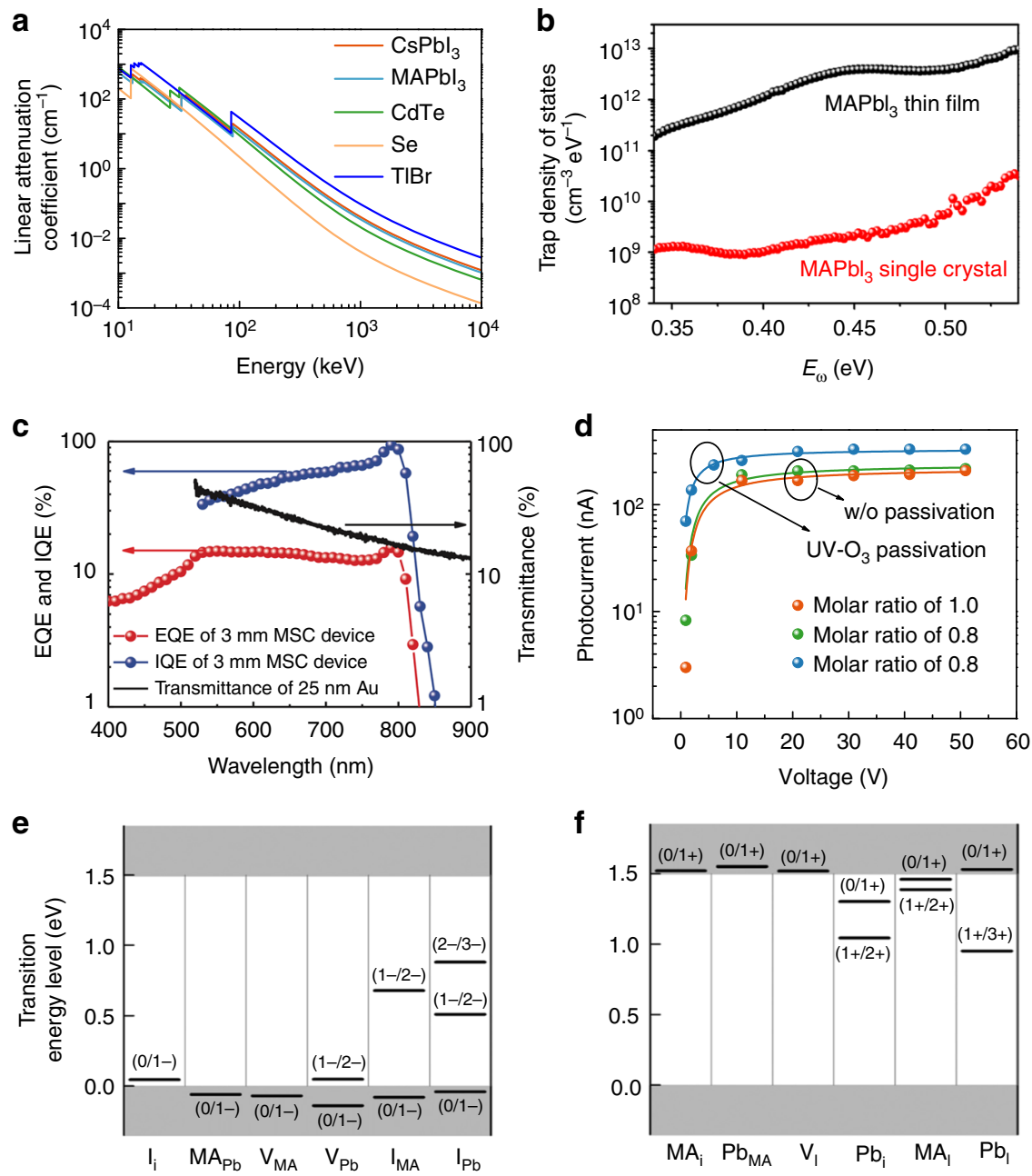

Fig. 1 Electronic properties of halide perovskites. a Linear attenuation coefficient of $\mathrm{MAPbl}_{3}, \mathrm{MAPbBr}_{3}, \mathrm{CdTe}$, Se, and TIBr versus photons energy. $\mathbf{b}$ Trap density of states of the $\mathrm{MAPbl}_{3}$ single crystal (red dots) and the thin film (black dots) measured by thermal admittance spectroscopy at room temperature. c External quantum efficiency (EQE) and internal quantum efficiency (IQE) spectra of a 3-mm-thick $\mathrm{MAPbl}_{3}$ single crystal along with the transmittance of 25 -nm-thick gold electrode. $\mathbf{d}$ The $\mu \tau$ product of $\mathrm{MAPbBr}_{3}$ crystal devices with different raw materials feeding ratio and surface passivation procedure. The transition energy levels of $\mathbf{e}$ intrinsic acceptors and $\mathbf{f}$ intrinsic donors in $\mathrm{CH}_{3} \mathrm{NH}_{3} \mathrm{Pbl}_{3}$ perovskite. $\mathbf{b}, \mathbf{c}$ are adapted with permission from ref. ${ }^{43}$, AAAS. $\mathbf{d}$ is adapted with permission from ref. ${ }^{18}$, Springer Nature. $\mathbf{e}, \mathbf{f}$ are adapted with permission from ref. ${ }^{49}$, American Institute of Physics

of full-width at half-maximum value of the photopeak and its corresponding photon energy. The semiconductor detectors for $\gamma$-ray spectrum acquisition actually have been developed for several decades, while there are still only a handful of semiconductors that can be successfully applied for this purpose with satisfying performance ${ }^{30}$, since $\gamma$-ray detection imposes multiplestringent requirements on semiconductor materials. Due to the much higher energy of $\gamma$-ray than X-ray, it needs materials to possess large atomic number $(Z)$ for strong stopping power ${ }^{31}$; large electron and hole $\mu \tau E^{\prime}$ 'product (or Schubweg distance) for efficient charge collection, where $E^{\prime}$ is the applied electric field; a low density of trap states to avoid charge trapping or recombination during charge collection for single event analysis; again, a large bulk resistivity over $10^{9} \Omega \mathrm{cm}$ is paramount for high signal/ noise ratio.

Table 2 lists several figures of merit of typical $\gamma$-ray semiconductor detectors that can impact the energy spectrum resolution. HPGe-based spectrum detectors now gives the best energy resolution of $0.3 \%^{32,33}$, while it requires cryogenic cooling to suppress its dark current due to its small bandgap of $0.66 \mathrm{eV}$. CZT materials have a larger bandgap of over $1.6 \mathrm{eV}$, and its large $\mathrm{Z}$ elements ensure a strong stopping power. A significant amount of efforts have been devoted to increase the bulk resistivity to around $10^{9}$ to $10^{10} \Omega \mathrm{cm}$ by crystal growth. The state-of-the-art CZT detectors achieved an energy resolution of $0.5 \%$, which operates at room temperature ${ }^{34,35}$. $\mathrm{TlBr}$ is another semiconductor that makes detectors with energy resolution of about $1 \%$ at room temperature, because of its large stopping power (four to five times stronger than CZT), high resistivity of over $10^{11} \Omega \mathrm{cm}$, and large bandgap of $2.68 \mathrm{eV}$, although the best reported $\mu \tau$ product of this material is still one order of magnitude smaller than $\mathrm{CZT}^{36}$, resulting in a very good energy resolution of $1 \%$. Notably, high-quality CZT materials are also not broadly commercially available, and now CZT single crystals cost about $\$ 3000$ per $\mathrm{cm}^{3}$. While it is possible that the cost of CZT single crystals can be dramatically reduced by scaling up the manufacturing, industry is pushed back by the small demand of CZT materials in the radiation detection market, in strong contrast to silicon.

Similar to X-ray imaging, $\gamma$-spectrum can be also collected by a combination of scintillator and a sensitive pixel photodetector. Typical scintillators for $\gamma$-application are made of $\mathrm{NaI}(\mathrm{Tl})$ crystals and $\mathrm{CsI}(\mathrm{Tl})$ crystals. $\mathrm{NaI}(\mathrm{Tl})$ crystal emits $415 \mathrm{~nm}$ light with efficiency of $11.3 \%$, comparable to the $\mathrm{CsI}(\mathrm{Tl})$. The emitting light 
Table 1 Performance of conventional X-ray detectors for medical imaging

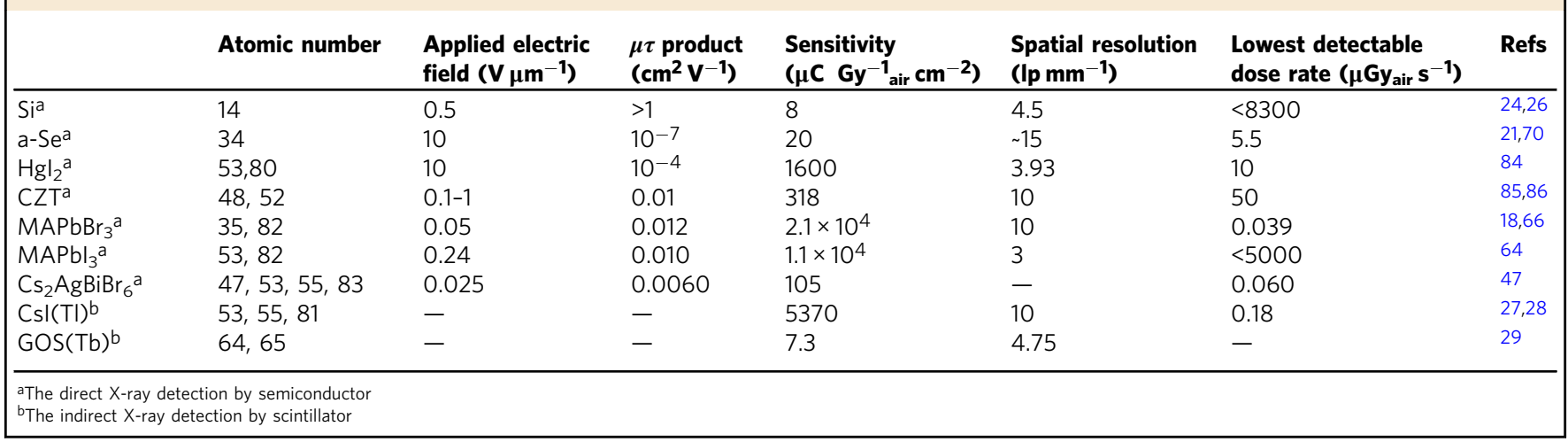

Table 2 Detailed $\gamma$-ray detector for energy spectrum

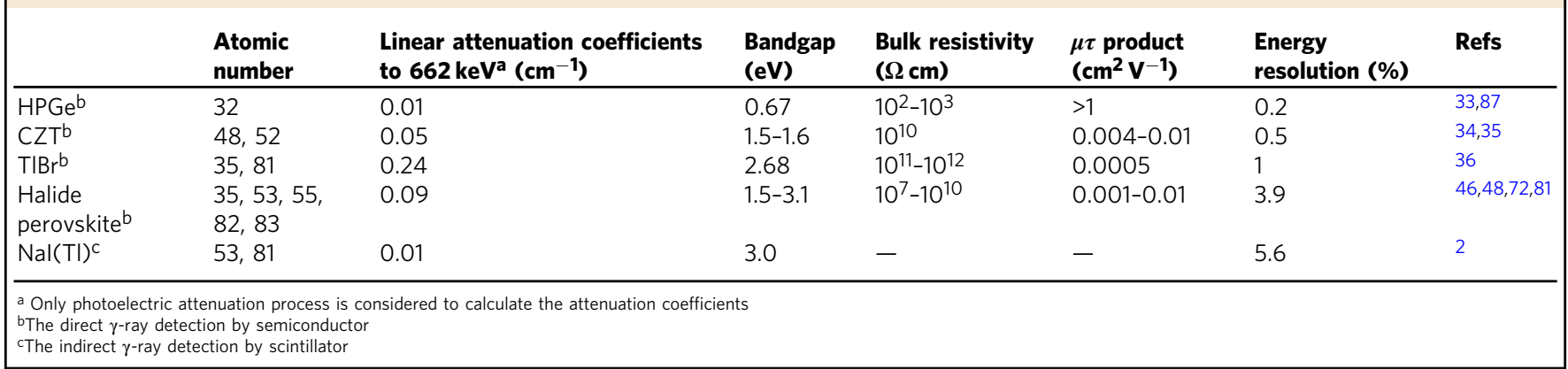

will be detected by a PMT detector to record light intensity, which is proportional to the $\gamma$-ray energy. Recent technology is moving toward $\gamma$-detector array so that spectrum can be quickly collected by multiple detectors, which however needs a really good uniformity of performance among all these $\gamma$-ray pixels.

\section{Halide perovskite materials emerge as promising candidates for radiation detectors}

Right after the demonstration of efficient solar cell application, halide perovskites were shown to be good photodetector materials due to the large light absorption coefficient, tunable bandgap, large mobility, and long carrier recombination lifetime, even in the form of polycrystalline films made from solution ${ }^{37-40}$. All these properties are actually also desired for radiation detection. In this section, we review the properties of perovskites, which make them a new generation of radiation detection materials.

Strong stopping power. In halide perovskites, the atomic number of $\mathrm{Cs}^{+}, \mathrm{Pb}^{2+}, \mathrm{Ag}^{+}, \mathrm{Bi}^{3+}, \mathrm{Sn}^{2+}, \mathrm{I}^{-}$, and $\mathrm{Br}^{-}$ions are as high as 55, $82,47,83,50,53$, and 35 , respectively. Some of the atomic numbers are even larger than those of CZT as shown in Table 2. The typical $\mathrm{MAPbI}_{3}$ halide perovskite has a density of around $4 \mathrm{~g} / \mathrm{cm}^{3}$, providing a large linear attenuation coefficient of $10 \mathrm{~cm}^{-1}$ to $100 \mathrm{keV}$ $\mathrm{X}$-rays, which is comparable to that of CZT material as shown in Fig. 1a and Table 2. Inorganic halide perovskite $\mathrm{CsPbI}_{3}$ has a larger linear attenuation coefficient of $14 \mathrm{~cm}^{-1}$. The capability to form double perovskites, such as $\mathrm{Cs}_{2} \mathrm{AgBiI}_{6}$ and $\mathrm{Cs}_{2} \mathrm{AgBiBr}_{6}$, allows the incorporation of many other high $Z$ elements into the perovskite structure.

Large mu-tau product. Halide perovskites were first applied in dye-sensitized solar cell structure as dye on mesoporous $\mathrm{TiO}_{2}$ surface $^{41}$, while researchers later found that halide perovskite solar cells with planar heterojunction structure works well due to its long charge carrier diffusion length of over $1000 \mathrm{~nm}^{42}$, corresponding to a mu-tau $(\mu \tau)$ product of $3.9 \times 10^{-7} \mathrm{~cm}^{2} \mathrm{~V}^{-1}$. This result was confirmed by Yakunin et al.'s ${ }^{17}$ work using perovskite polycrystalline films for X-ray detectors. Perovskite single crystal presents much lower trap density by removing the grain boundaries of polycrystals as shown in Fig. $1 \mathrm{~b}$ as well as improved crystallinity. The internal quantum efficiency (IQE) spectrum in Fig. 1c shows that the charge collection efficiency of a 3-mm-thick $\mathrm{MAPbI}_{3}$ single crystal is approaching $100 \%$, indicating an extremely long charge carrier diffusion length ${ }^{43}$. The mobility and charge recombination lifetime measurement results show a large $\mu \tau$ product of $1.2 \times 10^{-2} \mathrm{~cm}^{2} \mathrm{~V}^{-1}$, corresponding to a long diffusion length of $175 \mu \mathrm{m}$ as the low limit under strong illumination, which is consistent with photoconductivity measurement showing large $\mu \tau$ product of $\mathrm{MAPbBr}_{3}$ perovskite single crystal ${ }^{18}$, as shown in Fig. $1 \mathrm{~d}$. The $\mu \tau$ products of $\mathrm{MAPbI}_{3}$ and $\mathrm{MAPbBr}_{3}$ single crystals are comparable to that of CZT crystals, and are much larger than $\mathrm{TlBr}$ at room temperature, as shown in Table 2.

Large bulk resistivity. The bulk resistivity of the semiconductors is another important figure of merit in radiation detectors for small dark current and noise. One challenge in halide perovskite field is the difficulty in doping them intentionally, although this makes them attractive for radiation detection. Nevertheless, these materials are still subjected to self-doping. Halide perovskites showing excellent photovoltaic performance are mostly intrinsic or weakly $p$-type ${ }^{44}$. Replacing partial or all $\mathrm{I}^{-}$ions with $\mathrm{Br}^{-}$ions or $\mathrm{Cl}^{-}$ions can reduce the intrinsic charge carrier concentration due to enlarged bandgap. Typical $\mathrm{MAPbI}_{3}$ perovskite has a resistivity of around $10^{7} \Omega \mathrm{cm}$ at room temperature ${ }^{45}$, and the 
a

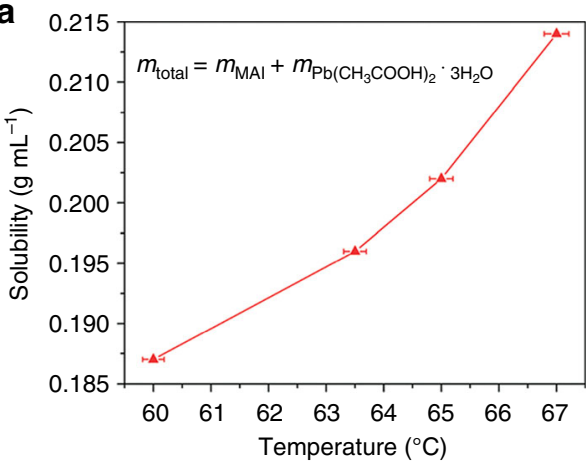

C

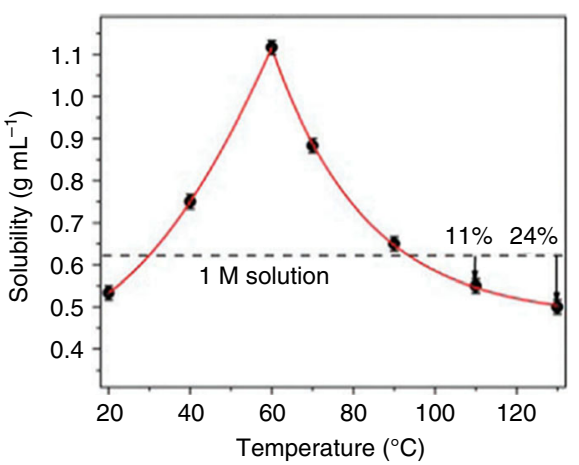

b

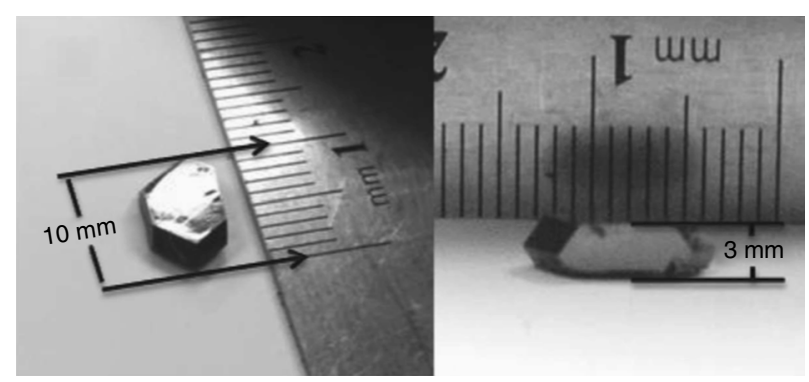

d
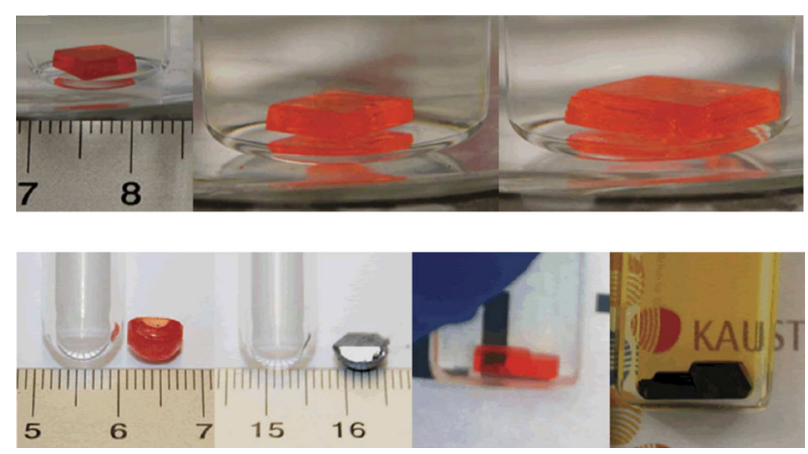

Fig. 2 Perovskite single crystals growth. a Solubility of $\mathrm{MAPbl}_{3}$ perovskite versus temperature in $\mathrm{HI}$ acid. $\mathbf{b}$ Photos of as-grown single crystal by top-seeded solution growth method. $\mathbf{c}$ Reverse solubility of $\mathrm{MAPbl}_{3}$ perovskite versus temperature in $\gamma$-butyrolactone (GBL). $\mathbf{d}$ Photos of as-grown halide perovskite single crystal by inverse temperature method. $\mathbf{a}$ is adapted with permission from ref. ${ }^{57}$, RSC Publishing. $\mathbf{b}$ is adapted with permission from ref. ${ }^{43}$, AAAS. $\mathbf{c}$ is adapted with permission from ref. ${ }^{58}$, American Institute of Physics. $\mathbf{d}$ is adapted with permission from ref. 59 , Springer Nature

resistivity of $\mathrm{MAPbBr}_{3}$ perovskite can be normally ten-folds higher ${ }^{46}$. These resistivity are still much smaller than those calculated from intrinsic charge concentration, indicating an unintentional doping in these materials, which was reported ${ }^{44}$. While the resistivity of inorganic halide perovskites can reach $10^{10} \Omega$ $\mathrm{cm}$, satisfying the requirement for $\gamma$-ray spectroscopy, but the mobility or $\mu \tau$ product is much lower than CZT materials ${ }^{47,48}$ (See Table 2).

Radiation hardness. One unique property of halide perovskites is its relatively better tolerance to defects than other semiconductor materials. First-principle calculation shows that the deep charge traps in $\mathrm{MAPbI}_{3}$ such as $\mathrm{I}_{\mathrm{Pb}}, \mathrm{I}_{\mathrm{MA}}, \mathrm{Pb}_{\mathrm{i}}$, and $\mathrm{Pb}_{\mathrm{I}}$ have too large formation energy to form. Most point defects either in the conduction or valence bands are shallow traps, such as $\mathrm{MA}_{\mathrm{i}}, \mathrm{V}_{\mathrm{Pb}}$, $\mathrm{MA}_{\mathrm{Pb}}, \mathrm{I}_{\mathrm{i}}, \mathrm{V}_{\mathrm{I}}$, and $\mathrm{V}_{\mathrm{MA}}{ }^{49}$ (see Figs 1e, f). The defect tolerance may explain the reported long electron-hole diffusion length in the single crystalline materials ${ }^{42,43}$. The defect tolerance also gives rise to the radiation damage tolerance of these materials ${ }^{50-52}$.

Lang et al. ${ }^{53}$ reported the stability of $\mathrm{MAPbI}_{3}$ solar cells under proton irradiation. A reference device with initial power conversion efficiency (PCE) of $12.1 \%$ has only $20 \%$ drop in current density, but with no voltage and fill factor loss after irradiation with $68 \mathrm{MeV}$ protons with dose up to $1.02 \times 10^{13} \mathrm{p} \mathrm{cm}$ -2 . It is also encouraging to find that the device has self-healing capability, that is, the current density of the device recovered to its initial value after shelf storage for 10 days $^{53}$. Chen et al. ${ }^{54}$ found that the Frenkel defects were generated by applying bias on perovskite, which may be related to the current density loss under irradiation, since the built-in electric field may also cause Frenkel defects. Self-annihilation of Frenkel defects is also confirmed when releasing the electric field, which is in consistent with the observed self-healing effect. Yang et al. ${ }^{55}$ also tested the stability of perovskite solar cells to $\gamma$-ray irradiation. The PCE barely drops after over $1500 \mathrm{~h}$ continuous $\gamma$-ray irradiation with total exposed dose of $23 \mathrm{kGy}$, while glass is significantly colored by $\gamma$ ray irradiation ${ }^{55}$. In striking contrast, $\mathrm{CZT}$ detectors lose their performance severely after total $30 \mathrm{kGy} \gamma$-ray exposure ${ }^{56}$, indicating the good $\gamma$-ray hardness of perovskite materials.

Low-cost raw materials and crystal growth. The raw materials for halide perovskite are nature abundant and low cost. The total material cost for $1 \mathrm{~cm}^{3}$ perovskite single crystal is about $\$ 2.65$ to $\$ 3.65$ based on raw material prices from suppliers such as SigmaAldrich. Considering the material cost generally reduces by 5 to 10 times after scaling up the production, we estimate that material cost of halide perovskite single crystals is $<\$ 0.3$ per $\mathrm{cm}^{3}$, which is 3 to 4 orders of magnitude cheaper than CZT crystals. The device fabrication cost by single crystal growth or polycrystalline films deposition process is also low, because they are all done by low-cost solution processes. Large and high-quality perovskite single crystals can be grown from low-cost solution processes ${ }^{57-60}$ at relatively low temperature (lower than $150^{\circ} \mathrm{C}$ ) compared to traditional radiation detector crystals such as HPGe and CZT, which are often grown at temperatures around the materials' melting points.

In hydrohalic acid, halide perovskites solubility often increases at higher temperatures 57 as shown in Fig. 2a, and single crystals with several millimeter size can be grown by gradually reducing the solution temperature as shown in Fig. 2b. While in some organic solvents such as dimethylformamide and $\gamma$-butyrolactone (GBL), halide perovskite has a reduced solubility at higher temperature (Fig. 2c), in striking contrast to many other materials. It was argued that the inverse solubility was caused by the protons as a result of solvent degradation under high temperature ${ }^{61}$, while exception to this 
a

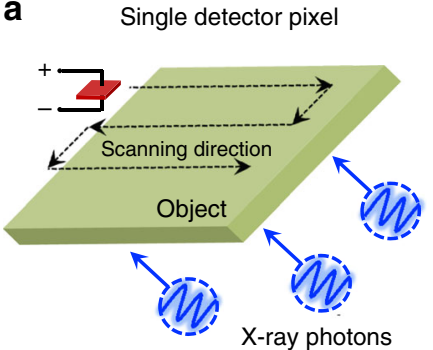

d

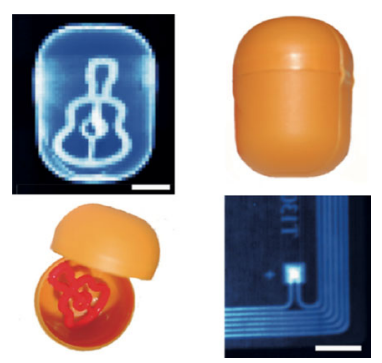

b

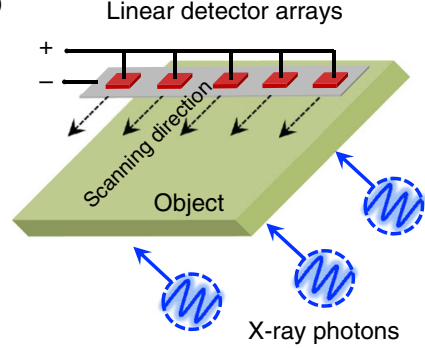

e

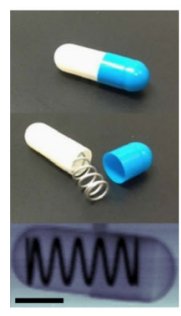

C
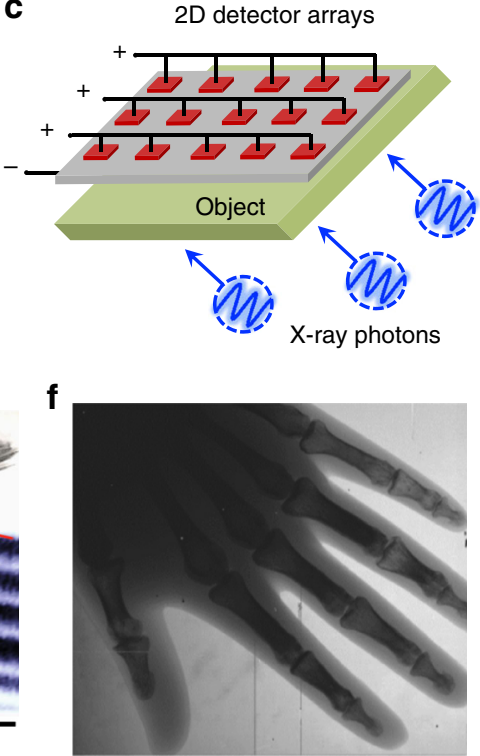

Fig. 3 X-ray imaging by single pixel, linear arrays, and two-dimensional (2D) arrays scanning. a-c Scheme of three different X-ray imaging processes: a single pixel scanning, $\mathbf{b}$ linear detector array (LDA) scanning, c 2D detector arrays scanning. $\mathbf{d}$ Optical and X-ray images of a plastic toy and an electronic key card. e Optical and X-ray images of an encapsulated metallic spring as well as a portion of a fish caudal fin (top, right) and X-ray image of a section of it (bottom, right). f A hand phantom X-ray image obtained from a muon piston calorimeter (MPC) detector (using $100 \mathrm{kVp}$ and $5 \mathrm{mGy} \mathrm{yir}^{-1}$ for $5 \mathrm{~ms}$ exposure, resulting in a dose of $25 \mu \mathrm{Gy}$ air and a bias voltage of $50 \mathrm{~V}$ ). Scale bars in $\mathbf{d}$ are $10 \mathrm{~mm}$ and the ones in e are $5 \mathrm{~mm} . \mathbf{d}\left(\right.$ ref. ${ }^{17}$ ), e (ref. $\left.{ }^{66}\right)$, and f $\left(\right.$ ref. ${ }^{64}$ ) are all adapted with permission from Springer Nature

rule can be easily named. The single crystal from such solvents can be grown by increasing the temperature from saturated solution ${ }^{58}$, and high-quality halide perovskite with dimension size over several millimeters can be obtained at a fast rate of $0.1-0.2 \mathrm{~cm} \mathrm{~h}^{-1}$ as illustrated in Fig. $2 \mathrm{~d}^{59}$. The measured hole carrier mobility of $\mathrm{MAPbI}_{3}$ single crystal grown from GBL solvent is about $67 \mathrm{~cm}^{2} \mathrm{~V}^{-1}$ $\mathrm{s}^{-1}$, over two times smaller than mobility of $164 \mathrm{~cm}^{2} \mathrm{~V}^{-1} \mathrm{~s}^{-1}$ of $\mathrm{MAPbI}_{3}$ single crystal grown from $\mathrm{HI}$ solvent ${ }^{43}$. This may be caused by the better quality of crystals grown from hydrohalic acid, since the crystal growth speed in hydrohalic acid is as slow as $0.2 \mathrm{~mm}$ per day. In addition, an anti-solvent approach has also been employed to grow perovskite single crystals from mixture solvents, which may give uniform growth rate of single crystals at room temperature ${ }^{62-64}$.

\section{Advance in the development of perovskite X-ray imaging detectors}

The first perovskite radiation detector made by $\mathrm{MAPbI}_{3}$ single crystal showed response to high-energy $\gamma$-ray photons under zero bias $^{43}$. The device worked in $\gamma$-voltaic mode with a quantum efficiency of $4 \%$ under high flux $\gamma$-ray. Later polycrystalline perovskite films made by spray coating were demonstrated to have good $\mathrm{X}$-ray response with imaging capability by moving a single pixel detector along $\mathrm{X}-\mathrm{Y}$ directions ${ }^{17}$. By scanning the signal current induced by transmitted X-ray photons, X-ray images of plastic target with clear inner scene were observed, as illustrated in Fig. 3a-d. The imaging time using single pixel detectors is very long, since it takes a lot of time to move the detector or the object along the $\mathrm{X}-\mathrm{Y}$ plane. Nevertheless, it is still a good conceptual demonstration for perovskite materials for Xray imaging application. Commercial X-ray detector products for imaging often employ linear detector arrays (LDAs), or, more frequently, flat panel arrays for fast detection. LDAs composed of individual X-ray-sensitive materials are arranged in a line as linear pixels as shown in Fig. $3 \mathrm{~b}$, which can scan along one direction to finish imaging (Fig. 3e). Flat panel arrays (Fig. 3c), which are often constructed by integrating X-ray-sensitive semiconductors on application-specific integrated circuits like CMOS read-out circuitry, can be employed to map an object by detecting the spatially dependent transmitted photon flux ${ }^{10}$. In this section, we review advancements in the development of perovskite single crystals and polycrystalline films for X-ray detection in the form of single pixel, LDAs, and two-dimensional (2D) detector arrays.

Single pixel perovskite detectors. Due to the large atomic number and material density of halide perovskite, the linear attenuation coefficient of $\mathrm{MAPbI}_{3}$ to $120 \mathrm{keV} \mathrm{X}$-rays is about 10 times stronger than $\alpha$-Se. Nevertheless, $\mathrm{MAPbBr}_{3}$ with slightly weaker X-ray stopping power has been more often used for X-ray detection, because of many advantages including simple single crystal growth, cubic crystal shape, large bandgap, easily crystal quality inspection due to its transparency in visible light range, and less ion migration. One issue frequently encountered is that the $\mathrm{MAPbBr}_{3}$ single crystals are not transparent with visible defects, which can be identified by the naked eye. Highly transparent $\mathrm{MAPbBr}_{3}$ single crystals are grown using precursor solution with non-stoichiometry precursor ratio. Since the $\mathrm{PbBr}_{2}$ has less solubility and precipitates faster than $\mathrm{MABr}$ in perovskite solution, a $\mathrm{PbBr}_{2} / \mathrm{MABr}$ feeding ratio of 0.8 can get equal precursor precipitating speed, which allows the growth of highquality single crystals. The $\mu \tau$ product of such $\mathrm{MAPbBr}_{3}$ single crystals reached $1.4 \times 10^{-2} \mathrm{~cm}^{2} \mathrm{~V}^{-1}$ (ref. ${ }^{18}$ ). Moreover, $\mathrm{Cl}^{-}$ions alloying can further improve the hole mobility to 560 $\mathrm{cm}^{2} \mathrm{~V}^{-1} \mathrm{~s}^{-1}$ (ref. ${ }^{46}$ ), which increased $\mu \tau$ product to $1.8 \times 10^{-2} \mathrm{~cm}^{2} \mathrm{~V}^{-1}$. In addition to $\mu \tau$ product, the surface charge recombination rate $(s)$ at perovskite/electrode interface also determines the signal intensity of an X-ray detector, and its dependence can be described by modified Hecht's equation:

$$
I=\frac{I_{0} \mu \tau V}{L^{2}} \frac{1-\exp \left(-\frac{L^{2}}{\mu \tau V}\right)}{1+\frac{L}{V} \frac{s}{\mu}},
$$


where $I$ is the measured photocurrent, $I_{0}$ is the saturated photocurrent, $L$ is the thickness, and $V$ is the applied bias. Clearly, a smaller $s$ can also increase photocurrent. One attractive property of $\mathrm{MAPbBr}_{3}$ in this context lies its automatic surface passivation by oxygen or moisture in air. The charge radiative recombination rate on the surface of $\mathrm{MAPbBr}_{3}$ single crystals varied by over two orders of magnitude upon physisorption or desorption of oxygen and water molecules ${ }^{65}$. Full physisorption of oxygen on $\mathrm{MAPbBr}_{3}$ surface resulted in a low surface recombination velocity of $4 \mathrm{~cm} \mathrm{~s}^{-1}$ in this material, even better than that well-passivated single crystalline silicon. In real operational devices, UV-ozone treatment of $\mathrm{MAPbBr}_{3}$ surface is a good strategy to retain the oxygen passivation effect, which successfully suppressed the surface recombination and resulted in a high X-ray sensitivity of $80 \mu \mathrm{CGy}^{-1}$ air $\mathrm{cm}^{-2}$ for the detector under a small bias of $0.1 \mathrm{~V}^{18}$. This sensitivity is already four times higher than $\alpha$-Se X-ray detectors as shown in Table $1^{21}$.

After demonstrating the superior detection properties of $\mathrm{MAPbBr}_{3}$ single crystals, a further step was taken to integrate them onto silicon, glass and other metallic substrates, which is touchstone to evaluate integration capability of perovskites with CMOS read-out circuitry. $\mathrm{MAPbBr}_{3}$ can be grown in solution at low temperatures below $120^{\circ} \mathrm{C}$, which is compatible with CMOS read-out circuitry. Wei et al. ${ }^{66}$ used a small-molecule of (3aminopropyl)triethoxysilane layer, which has a $\mathrm{NH}_{3} \mathrm{Br}$ terminate on one end and Si-O bond on the other end, to mechanically and electrically connect the $\mathrm{MAPbBr}_{3}$ single crystals with any surface that can bind with $\mathrm{Si}-\mathrm{O}$ bond ${ }^{66}$. The dipole of this bonding molecule at the interface of perovskite and $\mathrm{Si}$ also suppress the noise current while maintaining the signal current, which allows the device to operate under a larger optimal bias of $-7 \mathrm{~V}$. The large gain at larger bias made the Si-integrated $\mathrm{MAPbBr}_{3}$ single crystal detectors to have a much larger sensitivity of $2.1 \times 10^{4}$ $\mu \mathrm{CGy}^{-1}$ air $\mathrm{cm}^{-2}$ under $8 \mathrm{keV}$ soft X-ray. The lowest detectable dose rate was also further reduced down to $36 \mathrm{nGy}_{\mathrm{air}} \mathrm{s}^{-1}$, much better than the a-Se detectors as shown in Table 1 . $\mathrm{Cs}_{2} \mathrm{AgBiBr}_{6}$ single crystals have large resistivity over $10^{10} \Omega \mathrm{cm}$ despite small $\mu \tau$ product, which results in a detectable lowest $\mathrm{X}$-ray dose of $59.7 \mathrm{nGy}_{\text {air }} \mathrm{s}^{-1}$.

In addition to single crystals, polycrystalline perovskite bulk materials are formed by high-pressure sintering process from perovskite powders for X-ray detectors ${ }^{63}$. A polycrystalline perovskite wafer with diameter of $0.5 \mathrm{in}$. and thickness of $1 \mathrm{~mm}$ was demonstrated. Although the $\mu \tau$ product of the polycrystalline perovskite wafer was only $2 \times 10^{-4} \mathrm{~cm}^{2} \mathrm{~V}^{-1}$, about two orders of magnitude lower than that of the single crystals, a reasonably large sensitivity of $2527 \mu \mathrm{CGy}^{-1}$ air $\mathrm{cm}^{-2}$ was obtained under a relatively large electric field of $0.2{\mathrm{~V} \mu \mathrm{m}^{-1}}^{-1}$ (see Table 1). The challenge will be how to integrate them into CMOS read-out circuitry, because the high-pressure or high temperature process is often not compatible with CMOS technology.

Linear detector arrays. LDA scanning is more convenient than $2 \mathrm{D}$ arrays imaging in some cases where very large area $\mathrm{X}$-ray imaging needs to be performed by portable detectors like cargo inspection. The first perovskite LDA was demonstrated by depositing ten pixel electrodes in a row on a $\mathrm{MAPbBr}_{3}$ single crystal with a pixel electrode size of $200 \mu \mathrm{m}$ and a pitch of 400 $\mu \mathrm{m}$. The LDA devices by $\mathrm{MAPbBr}_{3}$ single crystal was demonstrated to possess good X-ray imaging capability at a very low dose rate of $36 \mathrm{nGy}_{\mathrm{air}} \mathrm{s}^{-1}$ (ref. ${ }^{66}$ ). Dose-area product (DAP) rate is a figure of merit used to compare absorbed doses in real medical imaging 67 . DAP rate of around $2.5 \mathrm{~Gy}_{\text {air }} \mathrm{cm}^{2} \mathrm{~h}^{-1}$ is estimated for perovskite detector in interventional cardiology with X-rays continuously on for capturing an image (entrance area of $100 \mathrm{~cm}^{2}$ ) of the human body, at least an order of magnitude better than that offered by state-of-the-art commercial medical radiographic systems ${ }^{68,69}$. The resolution of X-ray image reached $10 \mathrm{lp} \mathrm{mm}^{-1}$, which is comparable to those of the best CZT X-ray detectors and CsI(Tl) scintillator detectors as shown in Table 1 , and is still lower than $\alpha$-Se flat panel imagers ${ }^{70}$. In this case, the lateral diffusion of carriers seemed not to affect the resolution of these detector arrays, despite that the crystals was still thick, maybe due to the relatively large electric field applied.

2D detector arrays. Low temperature growth of perovskite single crystals makes it compatible with the CMOS read-out circuitry, and promising for high-sensitivity 2D X-ray detector arrays. The first 2D X-ray detector array was made of perovskite polycrystalline films ${ }^{64}$. Similar to $\alpha$-Se, polycrystalline perovskite films are actually attractive options for flat panel imagers, because they can be deposited directly on read-out circuitry with existing deposition techniques.

Doctor blading has been recently established to deposit halide perovskite films for solar cell modules with area close to $225 \mathrm{~cm}^{2}$ with module PCE of $\sim 15 \%{ }^{71}$. Spray coating is another mature technique to deposit thick films, and perovskite $\mathrm{X}$-ray pixel detectors were first demonstrated in 2015 by employing spraycoated $\mathrm{MAPbI}_{3}$ polycrystalline films with thickness of $60 \mu \mathrm{m}$, which is thick enough to detect soft $8 \mathrm{keV} \mathrm{X-rays}{ }^{17}$. Fullerene layers are often employed in these cases to block the pin holes to suppress leakage current ${ }^{37}$, and also passivate the surface charge traps on perovskites ${ }^{32}$. Recently, Kim et al. ${ }^{64}$ doctorbladed $830-\mu \mathrm{m}$-thick $\mathrm{MAPbI}_{3}$ films on a $10 \mathrm{~cm} \times 10 \mathrm{~cm}$ large TFT substrate ${ }^{64}$. Under irradiation from a $100 \mathrm{kVp}$ bremsstrahlung X-ray source, the 2D detector arrays presented good X-ray response under $50 \mathrm{~V}$ bias, showing clear X-ray image of a hand with bones observed inside in Fig. 3f. The image was acquired with $5 \mathrm{~ms}$ exposure of X-rays with dose rate of $5 \mathrm{mGy}_{\text {air }} \mathrm{s}^{-1}$, resulting in an exposed total dose of $25 \mu \mathrm{Gy} \mathrm{y}_{\text {air. }}$. The pixel size was around $70 \mu \mathrm{m} \times 70 \mu \mathrm{m}$, while the resolution was only $31 \mathrm{pmm}^{-1}$. This indicates that the resolution in these $2 \mathrm{D}$ arrays is still limited by the lateral charge diffusion among the crystal grains underneath neighboring pixel arrays, rather than the pixel size.

\section{Perovskite $\gamma$-ray detectors for photon energy spectrum}

In contrast to $\mathrm{X}$-rays, $\gamma$-rays have much higher energy and stronger penetrating capability. Fortunately, perovskite material has a strong stopping power to $\gamma$-rays with linear attenuation coefficient of $0.09 \mathrm{~cm}^{-1}$ at $662 \mathrm{keV}$ (photon energy of ${ }^{137} \mathrm{Cs}$ source), nearly two times larger than that of CdTe material ${ }^{46}$ (Fig. 1a and Table 2), and high-quality perovskite single crystals with centimeter size can be also easily grown from solution, which together enable sufficient stopping of $\gamma$-ray photons. Yakunin et al. ${ }^{72}$ firstly reported perovskite single crystal detectors toward $\gamma$-ray detection made by formamidinium lead triiodide $\left(\mathrm{FAPbI}_{3}\right)$ single crystals. The crystals are shown in the inset of Fig. $4 \mathrm{a}$, which have a large $\mu \tau$ product of $1 \times 10^{-2} \mathrm{~cm}^{2} \mathrm{~V}^{-1}$, and the devices showing good response to $59.6 \mathrm{keV}{ }^{241} \mathrm{Am} \gamma$-ray photons at room temperature (Fig. 4b). However, the black phase of $\mathrm{FAPbI}_{3}$ single crystal is not thermodynamically stable at room temperature. Higher energy $\gamma$-ray spectrum cannot be acquired in this case, since a larger crystal and larger bulk resistivity for the crystal are needed for stronger stopping power and smaller dark current, respectively.

Good $\gamma$-ray detectors should avoid the polarization effect, which describes the phenomenon of continuous dark current drift and 
a

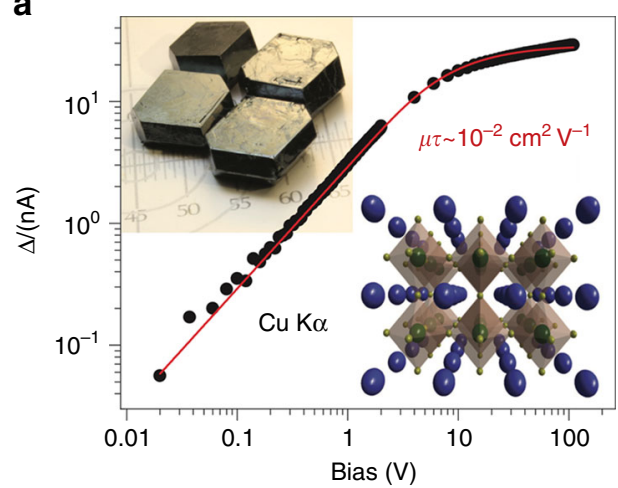

C

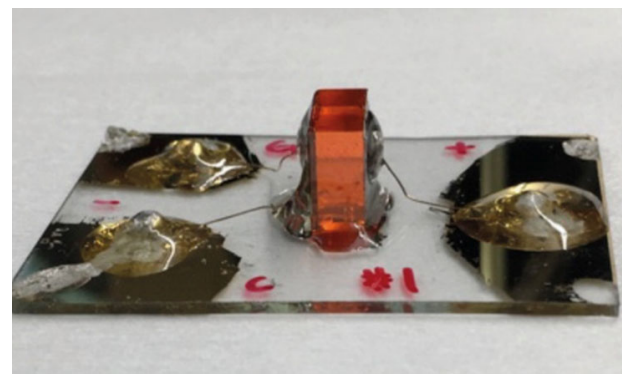

e
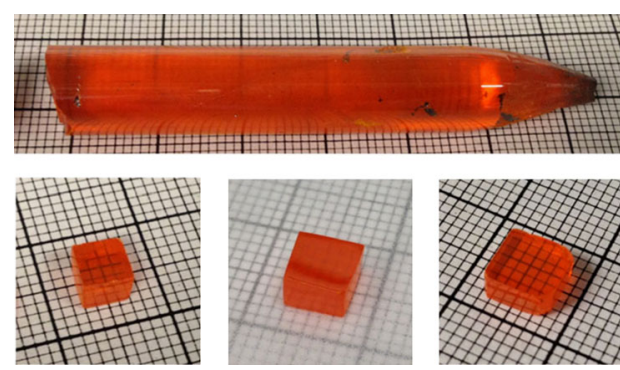

b

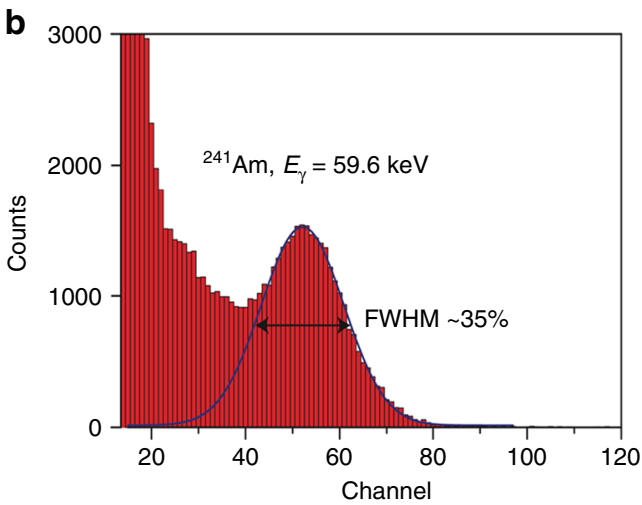

d

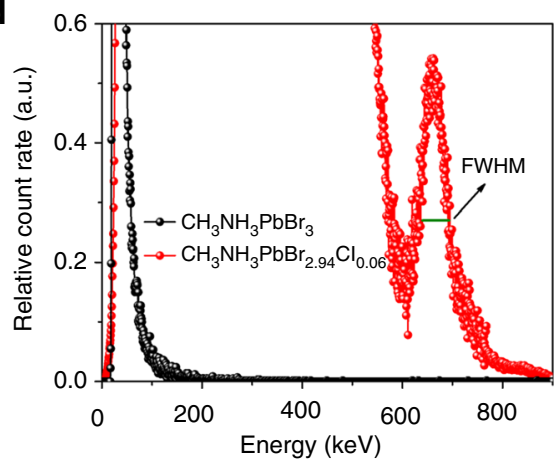

f

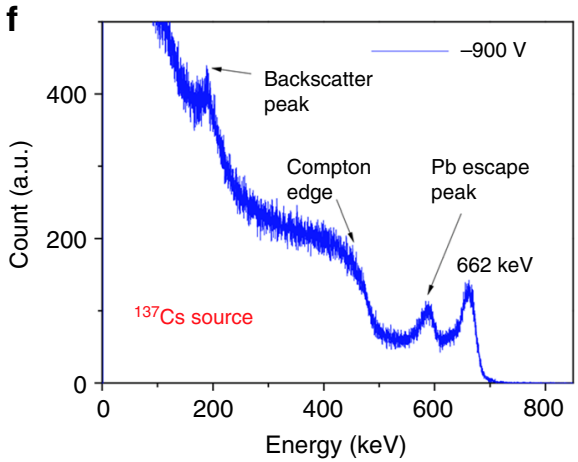

Fig. $4 \gamma$-Ray spectrum detection by halide perovskites. a The bias dependence of the photocurrent generated by $\mathrm{Cu} \mathrm{K \alpha} \mathrm{X-ray} \mathrm{in} \mathrm{a} \mathrm{MAPbl} 3$ single crystal (SC); the red line indicates a fit with the Hecht model. Top inset: Photograph of typical MAPbl 3 perovskite SCs grown from a non-aqueous method. Bottom inset: Schematic of the three-dimensional interconnection of $\mathrm{Pbl}_{6}$ octahedra in a perovskite lattice (green, $\mathrm{Pb}$; yellow, l; blue, MA). b Energy-resolved spectrum of ${ }^{241} \mathrm{Am}$ recorded with a $\mathrm{FAPb}_{3} \mathrm{SC}$. c Side view of a $\mathrm{CH}_{3} \mathrm{NH}_{3} \mathrm{PbBr}_{2.94} \mathrm{Cl}_{0.06} \mathrm{SC}$ detector, and electrode sides were encapsulated with epoxy. d Enlarged photopeak region of the ${ }^{137} \mathrm{Cs}$ energy spectrum obtained by $\mathrm{CH}_{3} \mathrm{NH}_{3} \mathrm{PbBr}_{2.94} \mathrm{Cl}_{0.06}$ and $\mathrm{CH}_{3} \mathrm{NH}_{3} \mathrm{PbBr}_{3} \mathrm{SC}$ detectors. e As-grown $\mathrm{CsPbBr} 3 \mathrm{SC}$ ingot with a diameter of $11 \mathrm{~mm}$, and the $\mathrm{SC}$ wafers with different sizes. $\mathbf{f}$ Energy-resolved spectrum of ${ }^{137} \mathrm{Cs} \gamma$-ray source with the characteristic energy of $662 \mathrm{keV}$ obtained by a $\mathrm{CsPbBr}_{3}$ detector. $\mathbf{a}, \mathbf{b}$ (ref. ${ }^{72}$ ), $\mathbf{c}, \mathbf{d}$ (ref. ${ }^{46}$ ), and $\mathbf{e}, \mathbf{f}\left(\right.$ ref. ${ }^{48}$ ) are all adapted with permission from Springer Nature

associated degradation of detector energy spectrum resolution ${ }^{73}$. The polarization effect is related to the ion migration in many $\gamma$-ray detecting semiconductors materials studied so far $^{74}$. The dark current stability and energy spectrum stability of device can be monitored to evaluate the degree of the polarization effect, which is also a very common method in traditional radiation detectors, such as $\mathrm{CZT}$ and $\mathrm{TlBr}$ single crystals ${ }^{73,75,76}$. Improving the bulk crystal quality and electrode contact can partially suppress the polarization effect at room temperature, although low temperature will also freeze the mobile ions ${ }^{77}$. Ion migration has been confirmed in halide perovskite materials, and the grain boundaries in polycrystalline films are shown to be the dominating ion migration channels $^{78}$. Nevertheless, polarization effect (or ion migration) can still occur in single crystals, depending on crystal quality, crystal composition, and applied electric field. For example, ion migration is often more notable in $\mathrm{I}^{-}$-based perovskite than $\mathrm{Br}^{-}$-based perovskites. Nazarenko et al. improved the $\mathrm{FAPbI}_{3}$ single crystal detector performance by alloying $\mathrm{Cs}^{+}$ions and $\mathrm{Br}^{-}$ions into bulk crystals to get mixture halide perovskite single crystal with composition of $\mathrm{Cs}_{x} \mathrm{FA}_{1-x} \mathrm{PbI}_{3-y} \mathrm{Br}_{y}(x=0-0.1, y=0-0.6)^{79}$. Since Cs + ions and $\mathrm{Br}^{-}$ions can partially stabilize the black phase of $\mathrm{FAPbI}_{3}$ single crystal, these have less ion migration compared to $\mathrm{FA}^{+}$ions and $\mathrm{I}^{-}$ions at room temperature, respectively ${ }^{80-82}$. However, despite that the $\mathrm{Cs}_{x} \mathrm{FA}_{1-x} \mathrm{PbI}_{3-y} \mathrm{Br}_{y}$ mixture perovskite single crystal showed $\mu \tau$ product of $0.12 \mathrm{~cm}^{2} \mathrm{~V}^{-1}$, neither the photopeak or the Compton edge were sharp or clear in the obtained spectrum $^{79}$. No reason was given in this work, but we suspect the low bulk resistivity and large device noise/dark current in this crystals lead to a low signal-to-noise ratio.

Dopant compensation is a good strategy to increase the resistivity of $\mathrm{MAPbBr}_{3}$ by alloying $n$-type $\mathrm{MAPbCl}_{3}$ into $p$-type $\mathrm{MAPbBr}_{3}$ to reduce its self-doping level ${ }^{46}$. The $\mathrm{MAPbBr}_{3}{ }_{x} \mathrm{Cl}_{x}$ 
perovskite single crystal gradually changed from $p$-type to $n$-type as increased $\mathrm{Cl}^{-}$ions amount. $\mathrm{MAPbBr}_{2.94} \mathrm{Cl}_{0.06}$ perovskite single crystal is almost intrinsic and has the lowest charge carrier concentration among all the alloys. The alloying technique also increased the $\mu \tau$ product from $1.2 \times 10^{-2}$ to $1.8 \times 10^{-2} \mathrm{~cm}^{2} \mathrm{~V}^{-1}$. By suppressing the crystal surface/edge leakage current with a guard ring electrode ${ }^{83}$ (Fig. 4c), a ten-fold improved bulk resistivity to $3.6 \times 10^{9} \Omega \mathrm{cm}$ is revealed for the $\mathrm{MAPbBr}_{2.94} \mathrm{Cl}_{0.06}$ perovskite single crystals ${ }^{46} .{ }^{137} \mathrm{Cs} \gamma$-ray energy spectrum with all features was then acquired at room temperature, benefiting from the high signal-to-noise ratio. The best photopeak energy resolution reached $6.5 \%$ as shown in Fig. $4 \mathrm{~d}$, as the first demonstration of $\gamma$ ray energy spectrum collection capability by halide perovskite single crystals ${ }^{46}$. Recently, $\mathrm{He}$ and co-workers improved the quality of $\mathrm{CsPbBr}_{3}$ single crystals grown from melt (Fig. 4e) by slowing down the crystal growth speed with a two-stage cooling process, first $20 \mathrm{~K} \mathrm{~h}^{-1}$ for $20 \mathrm{~h}$ and then $5 \mathrm{~K} \mathrm{~h}^{-1}$ for another $35 \mathrm{~h}$, which is critical parameter for crystal quality grown by high temperature Bridgman method ${ }^{48}$. Asymmetry electrodes of gold $(\mathrm{Au})$ and gallium $(\mathrm{Ga})$ serving as working electrodes on opposite surfaces of perovskite single crystals not only efficiently collect charges due to their work function difference ${ }^{43}$, the Schottky junction also suppressed device noise ${ }^{48}$. The crystals maintained a good $\mu \tau$ product of $1.34 \times 10^{-3} \mathrm{~cm}^{2} \mathrm{~V}^{-1} .{ }^{137} \mathrm{Cs} \gamma$-ray spectrum with $662 \mathrm{keV}$ photopeak was obtained with energy resolution of $3.9 \%$ using a thin crystal as shown in Fig. 4f, comparable to, or even better than, the commercial $\mathrm{NaI}(\mathrm{Tl}$ ) scintillator (See Table 2).

\section{Challenges and outlook}

Halide perovskites have been demonstrated as a new generation of room temperature radiation detection materials in the past 5 years. Efforts are still needed to improve the sensitivity of X-ray detectors for imaging, because the lowest detectable dose rate by the best reported perovskite detectors is still much higher than the average background irradiation of $0.1 \mathrm{nGy}_{\mathrm{air}} \mathrm{s}^{-1}$ in United States. Although increasing the working bias can enhance charge extraction in the detectors, it also increases noise. The dark current density of halide perovskite X-ray detectors is still higher than that of the commercial $\alpha$-Se detectors. Therefore, the resistivity of perovskites needs further improvement based on new material design and composition. The background charge carrier concentration is still dominated by the self-doping effect in halide perovskites, which limits the lowest detectable X-ray dose rate. Further improvement can be expected if deep mechanism of selfdoping is understood. Although these hybrid perovskite materials are more prone to degradation after exposure to heat or moisture than conventional detectors, it remains to be determined whether the environmental stability would impact the X-ray detectors, because perovskites will be encapsulated by X-ray penetration materials.

For spectroscopy applications, the state-of-the-art room temperature $\gamma$-ray detectors are made of CZT single crystals, which can achieve an energy resolution of around 0.5\%, although it takes several decades for them to reach this high resolution. The performance of halide perovskite $\gamma$-ray detectors is far from this level. The relatively lower bulk resistivity is among one of the challenges to be addressed. The size of high-quality single crystals is not large yet, though there should be no fundamental limit for achieving larger size. The competition between charge collection and charge recombination in these big crystals is not understood yet. Long-term continuous operation of halide perovskite devices under large field to detect X-rays and $\gamma$-rays is still a major concern, since the polarization effect may be still notable at room temperature. Designing a good perovskite X-ray imager or spectrometer should consider all the required material properties as discussed that lead to a high signal-to-noise ratio and fast response. Considering the nature-abundant and low-cost raw materials with all the exciting discoveries in such a short time, halide perovskites still hold a big promise to compete with traditional ionizing radiation detection materials.

Received: 24 November 2017 Accepted: 13 February 2019 Published online: 06 March 2019

\section{References}

1. Spahn, M. X-ray detectors in medical imaging. Nucl. Instrum. Meth. A 731, 57-63 (2013).

2. Carel, W. E. V. E. Inorganic scintillators in medical imaging. Phys. Med. Biol. 47, R85 (2002).

3. Duan, X. et al. X-ray cargo container inspection system with few-view projection imaging. Nucl. Instrum. Meth. A 598, 439-444 (2009).

4. Haff, R. P. \& Toyofuku, N. X-ray detection of defects and contaminants in the food industry. Sens. Instrum. Food Qual. 2, 262-273 (2008).

5. Chapman, H. N. et al. Femtosecond X-ray protein nanocrystallography. Nature 470, 73 (2011).

6. Harding, G. X-ray scatter tomography for explosives detection. Radiat. Phys. Chem. 71, 869-881 (2004).

7. Sales, B. C. \& Boatner, L. A. Lead-iron phosphate glass: a stable storage medium for high-level nuclear waste. Science 226, 45 (1984).

8. Yaffe, M. J. \& Rowlands, J. A. X-ray detectors for digital radiography. Phys. Med. Biol. 42, 1 (1997).

9. Zentai, G. X-ray imaging for homeland security. Int. J. Signal Imaging Syst. Eng. 3, 13-20 (2010).

10. Kasap, S. et al. Amorphous and polycrystalline photoconductors for direct conversion flat panel X-ray image sensors. Sensors 11, 5112 (2011).

11. Devanathan, R., Corrales, L. R., Gao, F. \& Weber, W. J. Signal variance in gamma-ray detectors-a review. Nucl. Instrum. Meth. A 565, 637-649 (2006)

12. Lin, E. C. Radiation risk from medical imaging. Mayo Clin. Proc. 85, 1142-1146 (2010)

13. Zheng, X. et al. Defect passivation in hybrid perovskite solar cells using quaternary ammonium halide anions and cations. Nat. Energy 2, 17102 (2017).

14. Xiao, Z. et al. Efficient perovskite light-emitting diodes featuring nanometresized crystallites. Nat. Photon 11, 108 (2017).

15. Bao, C. et al. Low-noise and large-linear-dynamic-range photodetectors based on hybrid-perovskite thin-single-crystals. Adv. Mater. 29, 1703209 (2017).

16. Zhao, J. et al. Strained hybrid perovskite thin films and their impact on the intrinsic stability of perovskite solar cells. Sci. Adv. 3, eaao5616 (2017)

17. Yakunin, S. et al. Detection of X-ray photons by solution-processed lead halide perovskites. Nat. Photon 9, 444 (2015).

18. Wei, H. et al. Sensitive X-ray detectors made of methylammonium lead tribromide perovskite single crystals. Nat. Photon 10, 333 (2016).

19. Heiss, W. \& Brabec, C. Perovskites target X-ray detection. Nat. Photon 10, 288 (2016).

20. Cunningham, I. A. \& Shaw, R. Signal-to-noise optimization of medical imaging systems. J. Opt. Soc. Am. A 16, 621-632 (1999).

21. Kasap, S. O. X-ray sensitivity of photoconductors: application to stabilized a-Se. J. Phys. D 33, 2853 (2000).

22. Lingren, C. L. \& Butler, J. F. Evaluating the performance of semiconductor radiation detectors through static charge analysis. IEEE Trans. Nucl. Sci. 45, 1723-1725 (1998)

23. Huda, W. \& Abrahams, R. B. X-ray-based medical imaging and resolution. Am. J. Roentgenol. 204, W393-W397 (2015).

24. Guerra, M. et al. Performance of three different Si X-ray detectors for portable $\mathrm{XRF}$ spectrometers in cultural heritage applications. J. Instrum. 7, C10004 (2012).

25. Arfelli, F. et al. Silicon X-ray detector for synchrotron radiation digital radiology. Nucl. Instrum. Meth. A 353, 366-370 (1994).

26. Rikner, G. \& Grusell, E. Effects of radiation damage on p-type silicon detectors. Phys. Med. Biol. 28, 1261 (1983).

27. Nagarkar, V. V. et al. Structured CsI(Tl) Scintillators for X-ray Imaging Applications. IEEE Trans. Nucl. Sci. 45, 492-496 (1998)

28. Starkenburg, D. J. et al. Thin film organic photodetectors for indirect X-ray detection demonstrating low dose rate sensitivity at low voltage operation. J. Appl. Phys. 122, 225502 (2017).

29. Büchele, P. et al. X-ray imaging with scintillator-sensitized hybrid organic photodetectors. Nat. Photon 9, 843 (2015). 
30. Sellin, P. J. Recent advances in compound semiconductor radiation detectors. Nucl. Instrum. Meth. A 513, 332-339 (2003).

31. Evans, R. D. \& Noyau, A. The Atomic Nucleus, Vol. 582 (McGraw-Hill, New York, 2015)

32. Cooper, R. J., Amman, M., Luke, P. N. \& Vetter, K. A prototype high purity germanium detector for high resolution gamma-ray spectroscopy at high count rates. Nucl. Instrum. Meth. A 795, 167-173 (2015).

33. Owens, A. \& Peacock, A. Compound semiconductor radiation detectors. Nucl. Instrum. Meth. A 531, 18-37 (2004).

34. Limousin, O. New trends in CdTe and CdZnTe detectors for X- and gammaray applications. Nucl. Instrum. Meth. A 504, 24-37 (2003).

35. Zhang, F. et al. Characterization of the H3D ASIC readout system and $6.0 \mathrm{~cm}^{3}$ 3-D position sensitive CdZnTe detectors. IEEE Trans. Nucl. Sci. 59, 236-242 (2012).

36. Hitomi, K., Kikuchi, Y., Shoji, T. \& Ishii, K. Improvement of energy resolutions in TlBr detectors. Nucl. Instrum. Meth. A 607, 112-115 (2009).

37. Fang, Y. \& Huang, J. Resolving weak light of sub-picowatt per square centimeter by hybrid perovskite photodetectors enabled by noise reduction. Adv. Mater. 27, 2804-2810 (2015).

38. De Wolf, S. et al. Organometallic halide perovskites: sharp optical absorption edge and its relation to photovoltaic performance. J. Phys. Chem. Lett. 5 , 1035-1039 (2014).

39. Dou, L. et al. Solution-processed hybrid perovskite photodetectors with high detectivity. Nat. Commun. 5, 5404 (2014).

40. García de Arquer, F. P., Armin, A., Meredith, P. \& Sargent, E. H. Solutionprocessed semiconductors for next-generation photodetectors. Nat. Rev. Mater. 2, 16100 (2017).

41. Kojima, A., Teshima, K., Shirai, Y. \& Miyasaka, T. Organometal halide perovskites as visible-light sensitizers for photovoltaic cells. J. Am. Chem. Soc. 131, 6050-6051 (2009).

42. Stranks, S. D. et al. Electron-hole diffusion lengths exceeding 1 micrometer in an organometal trihalide perovskite absorber. Science 342, 341 (2013).

43. Dong, Q. et al. Electron-hole diffusion lengths $>175 \mu \mathrm{m}$ in solution grown $\mathrm{CH}_{3} \mathrm{NH}_{3} \mathrm{PbI}_{3}$ single crystals. Science 347, 967 (2015).

44. Wang, Q. et al. Qualifying composition dependent $\mathrm{p}$ and $\mathrm{n}$ self-doping in $\mathrm{CH}_{3} \mathrm{NH}_{3} \mathrm{PbI}_{3}$. Appl. Phys. Lett. 105, 163508 (2014).

45. Pisoni, A. et al. Ultra-low thermal conductivity in organic-inorganic hybrid perovskite $\mathrm{CH}_{3} \mathrm{NH}_{3} \mathrm{PbI}_{3}$. J. Phys. Chem. Lett. 5, 2488-2492 (2014).

46. Wei, $\mathrm{H}$. et al. Dopant compensation in alloyed $\mathrm{CH}_{3} \mathrm{NH}_{3} \mathrm{PbBr}_{3-\mathrm{x}} \mathrm{Cl}_{\mathrm{x}}$ perovskite single crystals for gamma-ray spectroscopy. Nat. Mater. 16, 826 (2017).

47. Pan, W. et al. $\mathrm{Cs}_{2} \mathrm{AgBiBr}_{6}$ single-crystal $\mathrm{X}$-ray detectors with a low detection limit. Nat. Photon 11, 726-732 (2017).

48. He, Y. et al. High spectral resolution of gamma-rays at room temperature by perovskite $\mathrm{CsPbBr}_{3}$ single crystals. Nat. Commun. 9, 1609 (2018).

49. Yin, W.-J., Shi, T. \& Yan, Y. Unusual defect physics in $\mathrm{CH}_{3} \mathrm{NH}_{3} \mathrm{PbI}_{3}$ perovskite solar cell absorber. Appl. Phys. Lett. 104, 063903 (2014).

50. Yuan, Y. \& Huang, J. Ion migration in organometal trihalide perovskite and its impact on photovoltaic efficiency and stability. Acc. Chem. Res 49, 286-293 (2016).

51. Huang, J., Yuan, Y., Shao, Y. \& Yan, Y. Understanding the physical properties of hybrid perovskites for photovoltaic applications. Nat. Rev. Mater. 2, 17042 (2017).

52. Lang, F. et al. Influence of radiation on the properties and the stability of hybrid perovskites. Adv. Mater. 30, 1702905 (2017).

53. Lang, F. et al. Radiation hardness and self-healing of perovskite solar cells. Adv. Mater. 28, 8726-8731 (2016).

54. Chen, B. et al. Large electrostrictive response in lead halide perovskites. Nat. Mater. 17, 1020-1026 (2018).

55. Yang, S. et al. Organohalide lead perovskites: more stable than glass under gamma-ray radiation. Adv. Mater. 0, 1805547 (2018).

56. Zanarini, M. et al. Radiation damage induced by $2 \mathrm{MeV}$ protons in CdTe and CdZnTe semiconductor detectors. Nucl. Instrum. Meth. B 213, 315-320 (2004).

57. Dang, Y. et al. Bulk crystal growth of hybrid perovskite material $\mathrm{CH}_{3} \mathrm{NH}_{3} \mathrm{PbI}_{3}$. CrystEngComm 17, 665-670 (2015).

58. Saidaminov, M. I., Abdelhady, A. L., Maculan, G. \& Bakr, O. M. Retrograde solubility of formamidinium and methylammonium lead halide perovskites enabling rapid single crystal growth. Chem. Commun. 51, 17658-17661 (2015).

59. Saidaminov, M. I. et al. High-quality bulk hybrid perovskite single crystals within minutes by inverse temperature crystallization. Nat. Commun. 6, 7586 (2015).

60. Saidaminov Makhsud, I. et al. Inorganic lead halide perovskite single crystals: phase-selective low-temperature growth, carrier transport properties, and self-powered photodetection. Adv. Opt. Mater. 5, 1600704 (2016).
61. Nayak, P. K. et al. Mechanism for rapid growth of organic-inorganic halide perovskite crystals. Nat. Commun. 7, 13303 (2016).

62. Shi, D. et al. Low trap-state density and long carrier diffusion in organolead trihalide perovskite single crystals. Science 347, 519 (2015).

63. Shrestha, S. et al. High-performance direct conversion X-ray detectors based on sintered hybrid lead triiodide perovskite wafers. Nat. Photon 11, 436 (2017).

64. Kim, Y. C. et al. Printable organometallic perovskite enables large-area, lowdose X-ray imaging. Nature 550, 87 (2017).

65. Fang, H.-H. et al. Ultrahigh sensitivity of methylammonium lead tribromide perovskite single crystals to environmental gases. Sci. Adv. 2, e1600534 (2016).

66. Wei, W. et al. Monolithic integration of hybrid perovskite single crystals with heterogenous substrate for highly sensitive X-ray imaging. Nat. Photon 11, 315 (2017).

67. Akinlade Bidemi, I., Farai Idowu, P. \& Okunade Akintunde, A. Survey of dose area product received by patients undergoing common radiological examinations in four centers in Nigeria. J. Appl. Clin. Med. Phys. 13, 188-196 (2012).

68. Davies, A. G. et al. Do flat detector cardiac X-ray systems convey advantages over image-intensifier-based systems? Study comparing X-ray dose and image quality. Eur. Radiol. 17, 1787-1794 (2007).

69. Viggiano, A. et al. Exposure reduction by optimization of the imaging toolchain in pulmonary vein isolation. Eur. Heart J. 34, P2344-P2344 (2013).

70. David, M. H., Belev, G., Kasap, S. \& Martin, J. Y. Measured and calculated Kfluorescence effects on the MTF of an amorphous-selenium based CCD x-ray detector. Med. Phys. 39, 608-622 (2012).

71. Chen, H. et al. A solvent- and vacuum-free route to large-area perovskite films for efficient solar modules. Nature 550, 92 (2017).

72. Yakunin, S. et al. Detection of gamma photons using solution-grown single crystals of hybrid lead halide perovskites. Nat. Photon 10, 585 (2016).

73. Leão, C. R. \& Lordi, V. Ionic current and polarization effect in TlBr. Phys. Rev. $B$ 87, 081202 (2013).

74. Luo, Y. et al. Direct observation of halide migration and its effect on the photoluminescence of methylammonium lead bromide perovskite single crystals. Adv. Mater. 29, 1703451-n/a (2017).

75. Schlesinger, T. E. et al. Cadmium zinc telluride and its use as a nuclear radiation detector material. Mater. Sci. Eng. R. Rep. 32, 103-189 (2001)

76. Del Sordo, S. et al. Progress in the development of CdTe and CdZnTe semiconductor radiation detectors for astrophysical and medical applications. Sensors 9, 3491-3526 (2009).

77. Du, M.-H. Effects of impurity doping on ionic conductivity and polarization phenomenon in TlBr. Appl. Phys. Lett. 102, 082102 (2013).

78. Shao, Y. et al. Grain boundary dominated ion migration in polycrystalline organic-inorganic halide perovskite films. Energy Environ. Sci. 9, 1752-1759 (2016).

79. Nazarenko, O. et al. Single crystals of caesium formamidinium lead halide perovskites: solution growth and gamma dosimetry. NPG Asia Mater. 9, e373 (2017).

80. McMeekin, D. P. et al. A mixed-cation lead mixed-halide perovskite absorber for tandem solar cells. Science 351, 151 (2016).

81. Stoumpos, C. C. et al. Crystal growth of the perovskite semiconductor $\mathrm{CsPbBr}$ : a new material for high-energy radiation detection. Cryst. Growth Des. 13, 2722-2727 (2013).

82. Dirin, D. N. et al. Solution-grown $\mathrm{CsPbBr}_{3}$ perovskite single crystals for photon detection. Chem. Mater. 28, 8470-8474 (2016).

83. Nakazawa, K. et al. Improvement of the CdTe diode detectors using a guardring electrode. IEEE Trans. Nucl. SCI 51, 1881-1885 (2004).

84. Zentai, G. et al. in Medical Imaging 200315 (SPIE). https://doi.org/10.1117/ 12.480227.

85. Ivanov, $\mathrm{Yu}, \mathrm{M}$. et al. The possibilities of using semi-insulating CdTe crystals as detecting material for X-ray imaging radiography. Phys. Stat. Sol. C 0, 840-844 (2003).

86. Bellazzini, R. et al. Chromatic X-ray imaging with a fine pitch CdTe sensor coupled to a large area photon counting pixel ASIC. J. Instrum. 8, C02028 (2013).

87. Yang, G. et al. Study on the properties of high purity germanium crystals. J. Phys. 606, 012013 (2015).

\section{Acknowledgements}

This work is financially supported by the Defense Threat Reduction Agency (Award No. HDTRA1-14-1-0030) and UNC Research Opportunities Initiative through the Center of Hybrid Materials Enabled Electronic Technology. We thank Dr. Seungman Yun from Rayence, Co. Ltd for reviewing and commenting some aspects of this paper. 


\section{Author contributions}

All authors contributed to write and review the manuscript.

\section{Additional information}

Competing interests: The authors declare no competing interests.

Reprints and permission information is available online at http://npg.nature.com/ reprintsandpermissions/

Journal peer review information: Nature Communications thanks the anonymous reviewers for their contribution to the peer review of this work.

Publisher's note: Springer Nature remains neutral with regard to jurisdictional claims in published maps and institutional affiliations. (c) (i) Open Access This article is licensed under a Creative Commons Attribution 4.0 International License, which permits use, sharing, adaptation, distribution and reproduction in any medium or format, as long as you give appropriate credit to the original author(s) and the source, provide a link to the Creative Commons license, and indicate if changes were made. The images or other third party material in this article are included in the article's Creative Commons license, unless indicated otherwise in a credit line to the material. If material is not included in the article's Creative Commons license and your intended use is not permitted by statutory regulation or exceeds the permitted use, you will need to obtain permission directly from the copyright holder. To view a copy of this license, visit http://creativecommons.org/ licenses/by/4.0/.

(c) The Author(s) 2019 